“C 2015 IEEE. Personal use of this material is permitted. Permission from IEEE must be obtained for all other uses, in any current or future media, including reprinting/republishing this material for advertising or promotional purposes, creating new collective works, for resale or redistribution to servers or lists, or reuse of any copyrighted component of this work in other works." 


\title{
Network Coded Non-Binary LDGM Codes Based on Lattices for a Multi-Access Relay System
}

\author{
Yuanye $\mathrm{Ma}^{\dagger}$, Zihuai $\mathrm{Lin}^{\dagger}$, Jun $\mathrm{Li}^{\dagger}$, Guoqiang $\mathrm{Mao}^{\ddagger}$, and Branka Vucetic ${ }^{\dagger}$ \\ ${ }^{\dagger}$ The University of Sydney, Sydney, Australia, Email: \{yuanye.ma, zihuai.lin, jun.li1, branka.vucetic\}@ sydney.edu.au \\ ${ }^{\ddagger}$ The University of Technology, Sydney and National ICT Australia (NICTA), Australia, Email: g.mao@ieee.org
}

\begin{abstract}
In this paper, we propose a novel network coded non-binary low-density generator matrix (LDGM) code structure for a multi-access relay system, where multiple sources transmit lattice signals to a destination with the help of a relay. Specifically, we first develop a network coded non-binary LDGM code structure by jointly considering lattice-signal transmissions at the sources and the relay. Then we derive the achievable computation rate $(A C R)$ for the proposed system and on that basis optimize the key parameters in the proposed structure to maximize the ACR. Furthermore, we optimize the network coded non-binary LDGM codes based on lattices to approach the ACR. Simulation results show that the optimal setting of the parameters is consistent with that obtained from our analysis and the proposed code structure outperforms the designed reference scheme.
\end{abstract}

\section{INTRODUCTION}

Wireless network coding [1] has attracted significant attention among the research community for its spectral efficiency. An important category of wireless network coding is called nested codes [2], which enables a destination to decode all the sources' information from a network coded packet forwarded by a relay. The authors in [3] proposed a code structure that jointly combined the convolutional codes and the nested codes and designed a criterion to optimize the code profiles. However, the coding scheme is only applicable to binary codes and transmissions in orthogonal channels. Thus, the spectral efficiency is relatively low.

An evolutionary coding method, called high-dimensional lattice network codes, can provide a relatively large coding gain and increase the spectral efficiency [4]. In [5], the authors proposed a class of novel nested convolutional lattice codes, which is designed based on lattices to achieve a high spectral efficiency. However, the decoding complexity increases exponentially with the lattice dimensions. Since low-density generator matrix (LDGM) codes can be regarded as a special type of low-density parity-check (LDPC) codes with a linear encoding complexity, constructing lattices based on LDGM codes guarantees a manageable encoding/decoding complexity with a good error performance.

Based on the above observation, in this paper, we are interested in the design of network coded LDGM codes over a finite field with a high spectral efficiency and a low decoding complexity. Specifically, we construct novel nested non-binary LDGM codes based on lattices in a multi-access relay system. A potential application of the proposed code structure is

This work of Yuanye Ma was supported by China Scholarship Council and Norman I Price Supplementary Scholarship. to improve the instant messaging services (IMS) [6], i.e., MSN and Tencent QQ, by network coding multiple small packets together to obtain a single nested coded packet with a good error performance. Our contributions in this paper are summarized as follows. We first develop a nested nonbinary LDGM code structure by jointly considering latticesignal transmissions at the sources and at the relay. Then we derive the achievable computation rate (ACR) for the proposed system and optimize the key parameters in the proposed code structure to maximize the ACR. Furthermore, we optimize the nested non-binary LDGM codes based on lattices to approach the ACR with a designed low complexity decoder. Simulation results show that (1) the optimal setting of the parameters is consistent with that obtained from our analysis; (2) the proposed code structure performs $2 \mathrm{~dB}$ better than the reference scheme at an average symbol error rate of $10^{-4}$.

\section{SYSTEM MODEL}

We consider a multi-access relay system with $L$ sources, a single relay and a destination, as shown in Fig. 1, where the relay receives transmissions from the $L$ sources and forwards a network coded message to the destination.

The transmission process is conducted in two time slots. Let boldface lowercase and uppercase letters denote vectors and matrices, respectively. In the first time slot, messages are transmitted from their respective sources to the relay, i.e., $\mathbf{y}_{s r}=\sum_{\ell=1}^{L} h_{\ell} \mathbf{x}_{\ell}+\mathbf{z}_{s r}$, where $\mathbf{y}_{s r}$ is the received signal at the relay from all the sources, $h_{\ell}$ is the complex channel coefficient of the link between the $\ell$ th source and the relay, $\mathbf{x}_{\ell}$ is the transmitted message from the $\ell$ th source, and $\mathbf{z}_{s r}$ represents a vector of additive white Gaussian noise (AWGN) samples, in which each element is an AWGN with a zero mean and one-sided variance $\sigma^{2}$. The transmission power at the $\ell$ th source is subject to the constraint $\frac{1}{n} E\left[\left\|\mathbf{x}_{\ell}\right\|^{2}\right] \leq P$, where $n$ denotes the message length. In the second time slot, the network coded message is transmitted from the relay to the destination, i.e., $\mathbf{y}_{r d}=h_{r d} \mathbf{x}_{r}+\mathbf{z}_{r d}$, where $\mathbf{y}_{r d}$ is the received signal at the destination from the relay, $h_{r d}$ is the complex channel coefficient of the link between the relay and the destination, $\mathbf{x}_{r}$ is the transmitted message from the relay, and $\mathbf{z}_{r d}$ is a vector of AWGN samples, in which each element is an AWGN with a zero mean and one-sided variance $\sigma^{2}$. The transmission power at the relay is subject to the constraint $\frac{1}{n} E\left[\left\|\mathbf{x}_{r}\right\|^{2}\right] \leq P$. We define the SNR as $\gamma=P /\left(2 \sigma^{2}\right)$. 


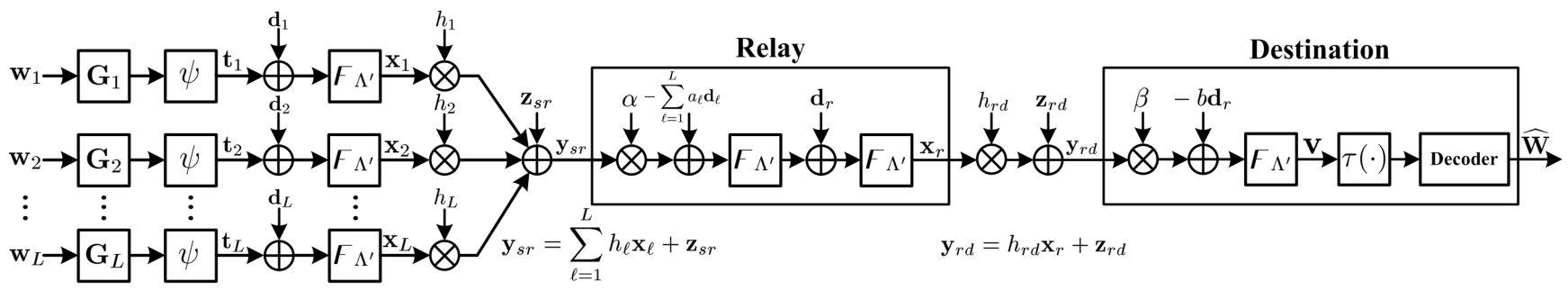

Fig. 1. A multi-access relay network model with multiple sources, one relay, and one destination.

\section{Nested LDGM Codes Based on Lattices}

In this section, we first present the mapping relationships between the values in a finite field and the points on a lattice. Then, we design the coding rules for the non-binary nested codes. At last, we elaborate the code structure of the nested LDGM codes based on lattices.

Let $\Lambda^{\prime}$ denote a coarse lattice, which is a subset of a fine lattice $\Lambda$, i.e., $\Lambda^{\prime} \subset \Lambda$. Let the message space be $\mathcal{W}=\Lambda / \Lambda^{\prime}$, where $\Lambda / \Lambda^{\prime}$ denotes the set of all the cosets of $\Lambda^{\prime}$ in $\Lambda$. Let $\mathbb{F}_{q}$ denote a finite field of size $q$, where $q$ is a positive prime integer and $q>2$. Let $\digamma_{q}(\mathbf{w})$ be an operation over $\mathbb{F}_{q}$ and let $\digamma_{\Lambda^{\prime}}(\boldsymbol{\lambda})$ be an operation over the fundamental Voronoi region of $\Lambda^{\prime}$ [4], denoted by $\mathcal{V}\left(\Lambda^{\prime}\right)$, where $\mathbf{w} \in \mathbb{F}_{q}$ and $\lambda \in \Lambda$. Thus,

$$
\begin{aligned}
& \digamma_{q}(\mathbf{w})=[\mathbf{w}] \bmod q, \digamma_{\Lambda^{\prime}}(\boldsymbol{\lambda})=[\boldsymbol{\lambda}] \bmod \Lambda^{\prime}, \\
& \digamma_{\Lambda^{\prime}}(\boldsymbol{\lambda})=\psi\left(\digamma_{q}(\mathbf{w})\right), \text { and } \digamma_{q}(\mathbf{w})=\psi^{-1}\left(\digamma_{\Lambda^{\prime}}(\boldsymbol{\lambda})\right),
\end{aligned}
$$

where $\psi(\cdot)$ denotes a map labeling the message over $\mathbb{F}_{q}$ to the points over $\mathcal{V}\left(\Lambda^{\prime}\right)$ and $\psi^{-1}(\cdot)$ denotes the inverse process [5].

Based on the bit-wised nested codes [2], we expand the coding field from the binary to $\mathbb{F}_{q}$. Let $\mathbf{w}_{\ell}$ denote the message generated independently and uniformly over $\mathbb{F}_{q}$ by the th source and let $\mathbf{G}_{\ell}$ denote the generator matrix over $\mathbb{F}_{q}$ at the $\ell$ th source. The mathematical operation of nested codes over $\mathbb{F}_{q}$ can be expressed by

$$
\digamma_{q}\left(\sum_{\ell=1}^{L} \mathbf{w}_{\ell} \mathbf{G}_{\ell}\right)=\digamma_{q}(\mathbf{W G}),
$$

where $\mathbf{W}=\left[\mathbf{w}_{1}, \mathbf{w}_{2}, \cdots, \mathbf{w}_{L}\right], \mathbf{G}=\left[\mathbf{G}_{1}^{T}, \mathbf{G}_{2}^{T}, \cdots, \mathbf{G}_{L}^{T}\right]^{T}$, $\mathbf{G}_{1}, \cdots, \mathbf{G}_{L}$ are mutually linearly independent generators at different sources, and $\mathbf{G}^{T}$ denotes the transpose of $\mathbf{G}$.

For the nested non-binary LDGM codes based on lattices, we regard the finite field $\mathbb{F}_{q}$ of the nested non-binary LDGM codes as the message space $\mathcal{W}$ of the lattices. Thus, the coded message $\mathbf{w}_{\ell} \mathbf{G}_{\ell}$ is uniformly distributed over an equivalent field with the message space $\mathcal{W}$. We define the message rate for each source to be the same as $R_{\ell} \triangleq \frac{1}{n} \log _{2}|\mathcal{W}|=r_{\ell} \log _{2} q$, where $r_{\ell}$ is the LDGM code rate at the $\ell$ th source.

At the $\ell$ th source, let $\mathbf{t}_{\ell}$ denote the coded message on $\Lambda$ and let $\mathbf{d}_{\ell}$ denote a dither generated independently according to a uniform distribution over $\mathcal{V}\left(\Lambda^{\prime}\right)$. Then we have

$$
\mathbf{t}_{\ell}=\psi\left(\mathbf{w}_{\ell} \mathbf{G}_{\ell}\right) \text { and } \mathbf{x}_{\ell}=\digamma_{\Lambda^{\prime}}\left(\mathbf{t}_{\ell}+\mathbf{d}_{\ell}\right) .
$$

At the relay, as in [4], we optimize the system performance by choosing some scale factor $\alpha$ and coefficient vector $\mathbf{a} \triangleq$ $\left(a_{1}, a_{2}, \cdots, a_{L}\right)$, where $\alpha \in \mathbb{C}, \mathbb{C}$ denotes the complex field, and $\mathbf{a} \in \Lambda$. It is worth noting that, to guarantee the successful decoding of all the messages at the destination, it is assumed that $a_{\ell} \neq 0, \forall \ell \in\{1,2, \cdots, L\}$. Then, we obtain

$$
\digamma_{\Lambda^{\prime}}\left(\alpha \mathbf{y}_{s r}-\sum_{\ell=1}^{L} a_{\ell} \mathbf{d}_{\ell}\right)=\digamma_{\Lambda^{\prime}}\left(\sum_{\ell=1}^{L} a_{\ell} \mathbf{t}_{\ell}+\mathbf{n}\right),
$$

where $\mathbf{n}=\sum_{\ell=1}^{L}\left(\alpha h_{\ell}-a_{\ell}\right) \mathbf{x}_{\ell}+\alpha \mathbf{z}_{s r}$.

Thus, the transmitted network coded message from the relay to the destination is $\mathbf{x}_{r}=\digamma_{\Lambda^{\prime}}\left(\sum_{\ell=1}^{L} a_{\ell} \mathbf{t}_{\ell}+\mathbf{n}+\mathbf{d}_{r}\right)$, where $\mathbf{d}_{r}$ is a dither generated independently according to a uniform distribution over $\mathcal{V}\left(\Lambda^{\prime}\right)$ at the relay.

At the destination, we remove the dither by choosing some scalars $\beta \in \mathbb{C}$ and $b \in \Lambda$, and obtain the received lattice signal $\mathbf{v}$ (see Fig. 1) given by

$$
\begin{aligned}
\mathbf{v} & =\digamma_{\Lambda^{\prime}}\left(\beta \mathbf{y}_{r d}-b \mathbf{d}_{r}\right)=\digamma_{\Lambda^{\prime}}\left(b \sum_{\ell=1}^{L} a_{\ell} \mathbf{t}_{\ell}+\mathbf{m}\right) \\
& =\digamma_{\Lambda^{\prime}}(\mathbf{u}+\mathbf{m}),
\end{aligned}
$$

where the effective noise $\mathbf{m}=b \mathbf{n}+\left(\beta h_{r d}-b\right) \mathbf{x}_{r}+\beta \mathbf{z}_{r d}$.

We then define a function $\tau(\cdot)$ that converts the received $i$ th lattice signal $v_{i}, v_{i} \in \mathbf{v}$ in (5), into the log-likelihood ratio vector $\mathbf{L}\left(u_{i}\right)$ as the input of the decoder, i.e.,

$$
\mathbf{L}\left(u_{i}\right)=\tau\left(v_{i}\right), \forall i \in\{1, \cdots, n\}, u_{i} \in \mathbf{u}, v_{i} \in \mathbf{v},
$$

where $\mathbf{L}\left(u_{i}\right) \triangleq\left[L\left(u_{i}=\lambda^{(1)}\right), \cdots, L\left(u_{i}=\lambda^{(q-1)}\right)\right]$. Let $\lambda^{(\xi)}$ denote the $\xi$ th non-zero fine lattice point in $\mathcal{V}\left(\Lambda^{\prime}\right)$, and we have $L\left(u_{i}=\lambda^{(\xi)}\right) \triangleq \ln \frac{\operatorname{Prob}\left(u_{i}=\lambda^{(\xi)}\right)}{\operatorname{Prob}\left(u_{i}=0\right)}, \forall \xi \in\{1, \cdots, q-1\}$.

The detailed process of $\tau(\cdot)$ function is given as follows. First, we expand (5), corresponding to the $i$ th signal, as

$$
\begin{aligned}
v_{i} & =\digamma_{\Lambda^{\prime}}\left(u_{i}+m_{i}\right) \\
& =\digamma_{\Lambda^{\prime}}\left(u_{i, r l}+j u_{i, i m}+m_{i, r l}+j m_{i, i m}\right) \\
& =\digamma_{\Lambda^{\prime}}\left(v_{i, r l}+j v_{i, i m}\right), m_{i} \in \mathbf{m},
\end{aligned}
$$

where $m_{i, r l}$ and $m_{i, i m}$ are approximated as the realizations of Gaussian random variables with the same variance $\sigma_{m}^{2}$. The joint probability density function of $v_{i, r l}, v_{i, i m}$ is

$$
\begin{array}{r}
p\left(v_{i} \mid u_{i}\right) \\
=\frac{1}{2 \pi \sigma_{m}^{2}} \sum_{k_{1}=-\infty}^{k_{1}=\infty} \sum_{k_{2}=-\infty}^{k_{2}=\infty} \exp \left(-\frac{\left(v_{i, r l}-u_{i, r l}-q k_{1}\right)^{2}}{2 \sigma_{m}^{2}}\right) \\
\times \exp \left(-\frac{\left(v_{i, i m}-u_{i, i m}-q k_{2}\right)^{2}}{2 \sigma_{m}^{2}}\right),
\end{array}
$$


where $q$ is the size of the finite field, as well as the number of fine lattice points over $\mathcal{V}\left(\Lambda^{\prime}\right)$.

Second, according to the Bayes' theorem, we can have

$$
\begin{aligned}
& \operatorname{Pr}\left(u_{i} \mid v_{i}\right)= \frac{p\left(v_{i} \mid u_{i}\right) \operatorname{Pr}\left(u_{i}\right)}{\operatorname{Pr}\left(v_{i}\right)} \\
&=g_{i} \sum_{k_{1}=-\infty}^{k_{1}=\infty} \sum_{k_{2}=-\infty}^{k_{2}=\infty} \exp \left(-\frac{\left(v_{i, r l}-u_{i, r l}-q k_{1}\right)^{2}}{2 \sigma_{m}^{2}}\right) \\
& \quad \times \exp \left(-\frac{\left(v_{i, i m}-u_{i, i m}-q k_{2}\right)^{2}}{2 \sigma_{m}^{2}}\right),
\end{aligned}
$$

where $g_{i}=\operatorname{Pr}\left(u_{i}\right) /\left(2 \pi \sigma_{m}^{2} \operatorname{Pr}\left(v_{i}\right)\right)$ is a constant if all the signals in $\mathcal{V}\left(\Lambda^{\prime}\right)$ are transmitted with equal probability. The normalized constant $g_{i}$ ensures $\operatorname{Pr}\left(u_{i}=0 \mid v_{i}\right)+\sum_{\xi=1}^{q-1} \operatorname{Pr}\left(u_{i}=\right.$ $\left.\lambda^{(\xi)} \mid v_{i}\right)=1$. Based on (9), we have the function $\tau(\cdot)$ as

$$
\begin{aligned}
& \tau\left(v_{i}\right)=\mathbf{L}\left(u_{i}\right) \\
= & {\left[\ln \frac{\operatorname{Pr}\left(u_{i}=\lambda^{(1)} \mid v_{i}\right)}{\operatorname{Pr}\left(u_{i}=0 \mid v_{i}\right)}, \cdots, \ln \frac{\operatorname{Pr}\left(u_{i}=\lambda^{(q-1)} \mid v_{i}\right)}{\operatorname{Pr}\left(u_{i}=0 \mid v_{i}\right)}\right] . }
\end{aligned}
$$

Hence, given the assumption that the destination knows all the assigned generators a priori and can obtain a sequence of corresponding coefficients, according to (2), it can extract all the messages from all the sources. It should be noticed that the proposed code structure is significantly different with that in [4]. Following the analysis in [4], only with sufficiently linear combinations of the transmitted signals sent from multiple relays, the destination can decode the messages individually. However, due to the joint design of the nested LDGM codes and lattices, the destination in our proposed code structure can decode all the messages from one network coded signal forwarded by the relay. Interested readers can refer to [3], [5] for further information regarding such a nested code structure.

\section{Achievable Computation Rate (ACR)}

In this section, we will analyze the ACR and optimize the key parameters $\alpha, \mathbf{a}, \beta$ and $b$ in the proposed code structure to maximize the ACR. The ACR is defined as follows: a message rate $R_{\ell}$ is said to be achievable if and only if for any $\epsilon>0$ and $n$ large enough, the destination can recover all the messages with an average probability of error $\epsilon$.

Theorem 1. For the complex-valued channels, the ACR of the proposed system can be expressed by

$$
\begin{aligned}
& \mathcal{R}\left(\mathbf{h}, \mathbf{a}, h_{r d}, b\right)= \\
& \log ^{+}\left(\frac{\gamma}{|b|^{2}\|\alpha \mathbf{h}-\mathbf{a}\|^{2} \gamma+|b|^{2}|\alpha|^{2}+\left|\beta h_{r d}-b\right|^{2} \gamma+|\beta|^{2}}\right),
\end{aligned}
$$

where $\log ^{+}(x) \triangleq \max \left(\log _{2}(x), 0\right)$.

Proof: Let $\mathbf{h}=\left[h_{1}, \cdots, h_{\ell}, \cdots, h_{L}\right]$. The ACR is obtained based on the observation that the destination can decode the message with arbitrary coefficients $a$ and $b$ on $\Lambda / \Lambda^{\prime}$. Therefore, the message rate is within the ACR as

$$
R_{\ell}<\min _{\mathbf{a}, b \neq 0} \mathcal{R}\left(\mathbf{h}, \mathbf{a}, h_{r d}, b\right) .
$$

The effective noise observed at the destination is expressed by $\mathbf{m}=b \mathbf{n}+\left(\beta h_{r d}-b\right) \mathbf{x}_{r}+\beta \mathbf{z}_{r d}$. Thus, the average power of the effective noise is $N_{e}=E\left[\|\mathbf{m}\|^{2} \mid \mathbf{h}, h_{r d}\right]=|b|^{2} \| \alpha \mathbf{h}-$ $\mathbf{a} \|^{2} P+2 \sigma^{2}|b|^{2}|\alpha|^{2}+\left|\beta h_{r d}-b\right|^{2} P+2 \sigma^{2}|\beta|^{2}$. The rate that can be achieved by the lattice code is less than that in [4]

$$
R_{\ell}<\min _{\mathbf{a}, b \neq 0} \frac{1}{2} \log ^{+}\left(\frac{P}{G(\Lambda) 4 \pi e \sigma_{m}^{2}}\right),
$$

where $\sigma_{m}^{2}$ is the one-side variance of the effective noise $\mathbf{m}$, $G(\Lambda)$ is the normalized second moment of the lattice $\Lambda$ and $\lim _{N \rightarrow \infty} G\left(\Lambda^{(N)}\right)=\frac{1}{2 \pi e}$. With $\forall \delta>0$, as the dimensions $N$ is large enough, we have that $G(\Lambda) 2 \pi e<(1+\delta)$. Meanwhile, $2 \sigma_{m}^{2}$ converges to $N_{e}$. It follows that for $N$ large enough, $2 \sigma_{m}^{2}<(1+\delta) N_{e}$. Thus, by choosing $\delta$ small enough, for the complex-valued channels, the ACR is given by (11).

To maximize the ACR, the related parameters $\alpha, \mathbf{a}, \beta$ and $b$ are optimized by the following propositions.

Proposition 1. The parameters $\alpha$ and $\beta$ that maximize the ACR are given by

$$
\alpha=\frac{\mathbf{a} \gamma \mathbf{h}^{\mathrm{H}}}{\gamma\|\mathbf{h}\|^{2}+1} \text { and } \beta=\frac{b \gamma h_{r d}^{\mathrm{H}}}{\gamma\left|h_{r d}\right|^{2}+1},
$$

where $\mathbf{a}$ is chosen by a greedy approach to maximize the ACR and $h^{\mathrm{H}}$ denotes the Hermitian transpose of $h$.

Proof: First, we show that maximizing the ACR is equivalent to minimizing $N_{e}$. From (5), we have

$$
\digamma_{\Lambda^{\prime}}\left(b \sum_{\ell=1}^{L} a_{\ell} \mathbf{t}_{\ell}+\mathbf{m}\right)=\Theta-\mathcal{Q}_{\Lambda^{\prime}}(\Theta),
$$

where $\Theta=b \sum_{\ell=1}^{L} a_{\ell} \mathbf{t}_{\ell}+\mathbf{m}$ and the mapping $\mathcal{Q}_{\Lambda^{\prime}}(\Theta) \triangleq$ $\arg \min _{\boldsymbol{\lambda}^{\prime} \in \Lambda^{\prime}}\left\|\Theta-\boldsymbol{\lambda}^{\prime}\right\|$. Since $\mathcal{Q}_{\Lambda^{\prime}}(\Theta)$ is a point on the coarse lattice $\Lambda^{\prime}$ and $\psi^{-1}\left(\mathcal{Q}_{\Lambda^{\prime}}(\Theta)\right)=0$, we can regard $\mathcal{Q}_{\Lambda^{\prime}}(\Theta)$ as a regular shift of the signal. Hence, $\alpha$ and $\beta$ should be chosen to minimize $N_{e}$, which is equivalent to maximizing the ACR.

It is apparent that $N_{e}$ is jointly convex in $\alpha$ and $\beta$. By solving $\frac{\partial N_{e}}{\partial \alpha}=0$ and $\frac{\partial N_{e}}{\partial \beta}=0$, we can have (14).

Proposition 2. Given channel coefficient parameters $\mathbf{h}$ and $h_{r d}$, the ACR is maximized by choosing the lattice network coding coefficient $b$ to be the closest point to the origin on $\Lambda$.

Proof : Inserting the expressions of $\alpha$ and $\beta$ into (11), we have another expression of the ACR. Then maximizing $\mathcal{R}\left(\mathbf{h}, \mathbf{a}, h_{r d}, b\right)$ can be regarded as an equivalent to the following minimization problem,

$$
\min _{b \neq 0}\left\{|b|^{2}\|\mathbf{a}\|^{2}-\frac{|b|^{2} \gamma\left|\mathbf{h}^{\mathrm{H}} \mathbf{a}\right|^{2}}{1+\gamma\|\mathbf{h}\|^{2}}+|b|^{2}-\frac{|b|^{2} \gamma\left|h_{r d}^{\mathrm{H}}\right|^{2}}{1+\gamma\left|h_{r d}\right|^{2}}\right\} .
$$

By extracting $|b|^{2}$, as $b \in \Lambda$, it is optimum to choose $b$ as the closest point to the origin on the fine lattice $\Lambda$.

The ACR is derived and used as a theoretical limit for the optimization of the proposed codes. Here, different from [4], which only considers the optimization of parameters $\alpha$ and a at the relay, we consider a different system model and optimize all the parameters $\alpha, \mathbf{a}, \beta$ and $b$ by maximizing the ACR for the entire system at the destination. 


\section{Code Optimization}

To optimize the proposed nested non-binary LDGM codes based on lattices, we first develop a corresponding low complexity decoder. Note that a conventional low complexity decoding algorithm, such as the Fast Fourier Transform (FFT), requires that the size of the finite field should be a power of two. However, in the case of our proposed code structure, the size of the finite field $q$ is a prime. Therefore, we employ and expand the Extended Min-Sum algorithm in [7] to a Latticebased Extended Min-Sum (L-EMS) algorithm, where the size of the finite field is a prime. The initialized channel input of the L-EMS decoder is estimated on the lattice by (10) and the exchanged messages between variable nodes and check nodes are truncated vectors with a length $n_{m} \leq q$. With the L-EMS decoder, the computational complexity is dominated by $\mathcal{O}\left(n_{m} \log _{2}\left(n_{m}\right)\right)$, compared with that of the Belief Propagation (BP) algorithm denominated by $\mathcal{O}\left(q^{2}\right)$ [8].

We then optimize the proposed codes with the L-EMS decoder. It should be noted that the structure of the nested non-binary LDGM codes based on lattices is identical to that of a single "stacked" non-binary LDGM code based on lattices. In particular, we consider only the average column weight $\bar{w}_{c}$ of the single "stacked" non-binary LDGM code, because it is intractable to locate the optimal code among a huge irregular LDGM matrix set. For a given LDGM code rate, we will optimize $\bar{w}_{c}$ with respect to SNR under a certain symbol error rate. Formally, we write $\bar{w}_{c, o p t}=\arg \min _{\bar{w}_{c}}\left\{\gamma\left(\bar{w}_{c}\right)\right\}$. As stated in [9], simulation based approaches have to be used to solve this optimization problem. In this paper, a latticebased Monte Carlo method is employed, where the initialized channel input on lattices is estimated as (10).

\section{REFERENCE SCHEME}

This section presents a reference scheme based on amplify and forward (AF) protocol ${ }^{1}$ to compare with the proposed code structure. For the AF, the received signal at the relay is still (1) and the received signal at the destination can be written as

$$
\mathbf{y}_{d}=h_{r d} \mathcal{A}\left(\sum_{\ell=1}^{L} h_{\ell} \mathbf{x}_{\ell}+\mathbf{z}_{s r}\right)+\mathbf{z}_{r d},
$$

where $\mathcal{A}$ is the amplification factor given by $\mathcal{A}=\sqrt{\frac{\gamma}{\gamma\|\mathbf{h}\|^{2}+1}}$. Then, to successfully decode the messages at the destination, analogue to the proposed code structure, we introduce a scale factor $\rho \in \mathbb{C}$ as well as a coefficient vector $\mathbf{c} \triangleq$ $\left(c_{1}, c_{2}, \cdots, c_{L}\right)$, where $\mathbf{c} \in \Lambda$ and $c_{\ell} \neq 0$, and then obtain

$$
\digamma_{\Lambda^{\prime}}\left(\rho \mathbf{y}_{d}-\sum_{\ell=1}^{L} c_{\ell} \mathbf{d}_{\ell}\right)=\digamma_{\Lambda^{\prime}}\left(\sum_{\ell=1}^{L} c_{\ell} \mathbf{t}_{\ell}+\mathbf{n}_{A F}\right),
$$

where $\mathbf{n}_{A F}=\sum_{\ell=1}^{L}\left(\rho h_{r d} \mathcal{A} h_{\ell}-c_{\ell}\right) \mathbf{x}_{\ell}+\rho h_{r d} \mathcal{A} \mathbf{z}_{s r}+\rho \mathbf{z}_{r d}$.

Based on Proposition 1, the scale factor $\rho$ can be obtained as

$$
\rho=\frac{\mathcal{A} \mathbf{c} \gamma \mathbf{h}^{\mathrm{H}} h_{r d}^{\mathrm{H}}}{\mathcal{A}^{2} \gamma\|\mathbf{h}\|^{2}\left|h_{r d}\right|^{2}+\mathcal{A}^{2}\left|h_{r d}\right|^{2}+1},
$$

\footnotetext{
${ }^{1}$ Decode and forward (DF) protocol is not considered for comparison because, to the best knowledge of the authors, there is not feasible method except the proposed code structure can be implemented to decode the messages at the relay.
}

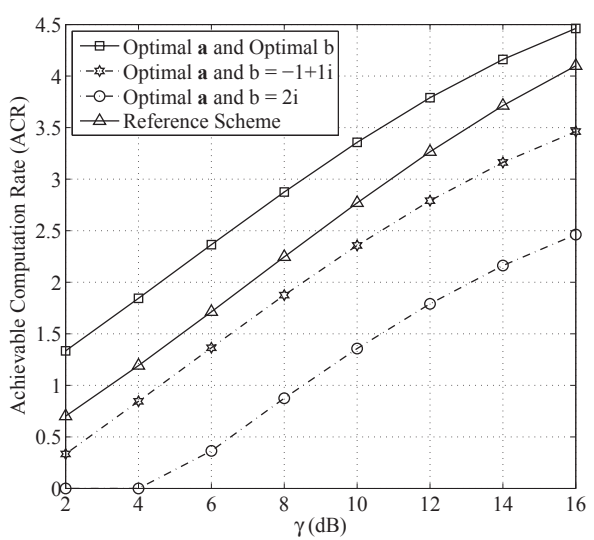

Fig. 2. The ACR performance of the proposed system with an optimal a and different choices of $b$, compared with that of the reference scheme.

where $\mathbf{c}$ can be readily obtained via a greedy approach. Thus, based on Theorem 1, the ACR of the reference scheme based on $\mathrm{AF}$, denoted by $\mathcal{R}_{A F}$, can be obtained as

$$
\mathcal{R}_{A F}=\log ^{+}\left(\frac{\gamma}{\left\|\rho h_{r d} \mathcal{A} \mathbf{h}-\mathbf{c}\right\|^{2} \gamma+|\rho|^{2}\left|h_{r d}\right|^{2} \mathcal{A}^{2}+|\rho|^{2}}\right) .
$$

\section{Numerical AND Simulation Results}

In the simulations, a multi-access relay system with two sources, one relay and one destination is considered. As in [10], the channels are set as $h_{1}=-1.17+2.15 j, h_{2}=1.25-$ $1.63 j$ and $h_{r d}=0.77+1.12 j$. The lattice partition is chosen to be a typical Gaussian integer $\mathcal{W} \cong \mathbb{Z}[\varpi] / \eta \mathbb{Z}[\varpi]$ as in [11], where $\eta=2+3 \varpi$. The finite field is $\mathbb{F}_{13}$. The LDGM code rate for each source is set as 0.25 and the LDGM code length is 2000. Thus the message rate $R_{\ell}=0.25 \log _{2} 13$. According to Propositions 1 and 2, the optimal lattice network coding coefficients $^{2}$ for the proposed code structure are computed by $\mathbf{a}=[-1,1]$ and $b=1$. For the reference scheme, we have $\mathbf{c}=[-1,1]$. Using the lattice-based Monte Carlo method, we obtain an optimal average column weight $\bar{w}_{c}=2.4$ for the proposed codes over $\mathbb{F}_{13}$. Subsequently, we construct the generator matrix $\mathbf{G}$ of the corresponding single "stacked" LDGM code. To assign each source with different linearly independent LDGM codes, we divide the generator matrix of the single "stacked" LDGM code as ${ }^{3}$

$$
\mathbf{G}^{1000 \times 2000}=\left[\begin{array}{l}
\mathbf{G}_{1}^{500 \times 2000} \\
\mathbf{G}_{2}^{500 \times 2000}
\end{array}\right] .
$$

Fig. 2 show the ACR performance of the proposed system with an optimal a and different choices of $b$, and that of the reference scheme is also plotted for comparison. It is

\footnotetext{
${ }^{2}$ Note that the optimal lattice network coding coefficients are the points on the fine lattice and there are multiple choices for optimal $\mathbf{a}$ or $b$ that maximize the ACR. Here we just present one optimal result of $\mathbf{a}$ or $b$.

${ }^{3}$ Here, we simulate symmetric case only where the rates for $\mathbf{G}_{1}$ and $\mathbf{G}_{2}$ are equal. However, it can be readily extended to asymmetric scenarios by assigning $\mathbf{G}_{1}$ and $\mathbf{G}_{2}$ with different rates.
} 


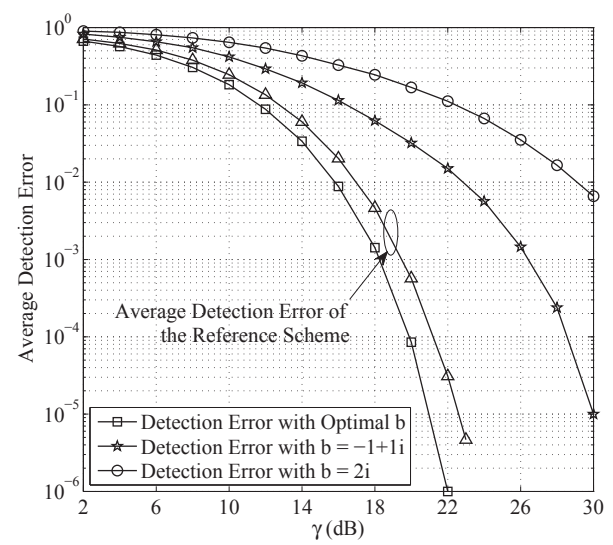

Fig. 3. The average detection error performance with different values of $b$.

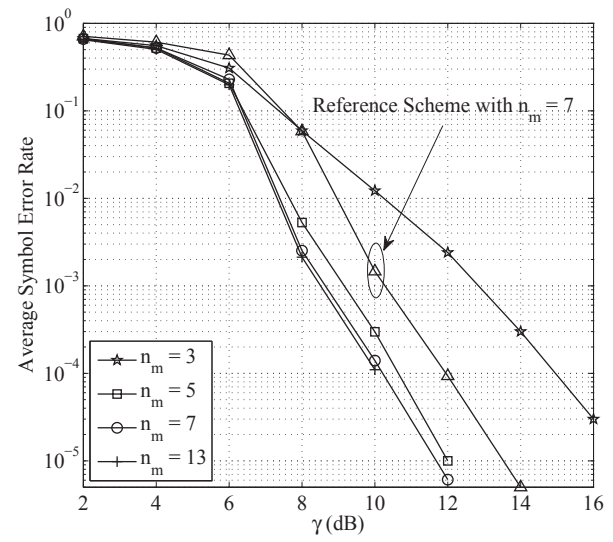

Fig. 4. The average symbol error performance of the proposed codes.

illustrated that the curve with the optimal $b$ achieves higher ACR than that with $b=-1+1 i$ and $b=2 i$, respectively, which validates the result in Proposition 2 that $b$ should be the closet point to the origin on $\Lambda$. It also can be observed that the proposed system with optimal lattice network coding coefficients performs better than the reference scheme.

Fig. 3 shows the average detection error performance of the proposed system with the lattice network coding coefficients chosen as $b=1, b=-1+1 i$, and $b=2 i$, respectively. Each curve hereafter is obtained by averaging over 1000 runs. The detection error is defined as the average symbol error rate at the input of the decoder (i.e., the output of the function $\tau(\cdot)$, see Fig. 1). Fig. 3 shows that the best average detection error performance is realized by choosing optimal $b=1$ compared with $b=-1+1 i$ and $b=2 i$, which is consistent with the result of Proposition 2 and the analysis for Fig. 2. Besides, the average detection error performance of the reference scheme is also shown for comparison, which reveals a worse performance than that of the proposed system.

In Fig. 4, the average symbol error performance of the optimized codes via Section V with the L-EMS decoder are depicted with the truncated message length $n_{m}=3,5,7$, and 13, respectively. A tradeoff exists between the code performance corresponding to the value of $n_{m}$ and the decoding complexity. It is shown that a further increase of $n_{m}$ from $n_{m}=7$ to 13 only results in a marginal performance improvement, which indicates that $n_{m}=7$ can be a good choice for the codes. Concerning the complexity, based on the analysis in Section V, the L-EMS decoder with $n_{m}=13$ is 2.44 times more complex than the L-EMS decoder with $n_{m}=7$. Furthermore, the performance of the reference scheme based on AF protocol with $n_{m}=7$ are also simulated in Fig. 4 for comparison. The reference scheme employs the same codes adopted by the proposed system. It is shown that the SNR required by the proposed code structure to achieve an average symbol rate of $10^{-4}$ is about $2 \mathrm{~dB}$ less than that required by the reference scheme to achieve the same error rate.

\section{CONCLUSION}

In this paper, we proposed novel nested non-binary LDGM codes based on lattices for a multi-access relay system. Specifically, we first constructed this novel codes by jointly considering lattice-signal transmissions at the sources and at the relay. Besides, we derived the ACR and optimized related parameters to maximize the ACR for the proposed system. Furthermore, we optimized the proposed codes by the latticebased Monte Carlo method to approach the ACR with the low complexity L-EMS decoder. Finally, simulation results showed that the optimal setting of the parameters is consistent with that suggested in our analysis and the proposed code structure performs $2 \mathrm{~dB}$ better than the reference scheme at an average symbol error rate of $10^{-4}$.

\section{REFERENCES}

[1] S. Katti, H. Rahul, W. Hu, D. Katabi, M. Médard, and J. Crowcroft, "Xors in the air: Practical wireless network coding," IEEE/ACM Trans. Networking, vol. 16, no. 3, pp. 497-510, Jun. 2008.

[2] L. Xiao, T. Fuja, J. Kliewer, and D. Costello, "Nested codes with multiple interpretations," in Proc. 40th Annual Conf. Inform. Sciences and Systems (CISS), Mar. 2006, pp. $851-856$.

[3] Y. Ma, Z. Lin, H. Chen, and B. Vucetic, "Multiple interpretations for multi-source multi-destination wireless relay network coded systems," in Proc. Int. Symp. Personal Indoor and Mobile Radio commun. (PIMRC), 2012, pp. 2253-2258.

[4] B. Nazer and M. Gastpar, "Compute-and-forward: Harnessing interference through structured codes," IEEE Trans. Inform. Theory, vol. 57, no. 10 , pp. $6463-6486$, Oct. 2011.

[5] Y. Ma, T. Huang, J. Li, J. Yuan, Z. Lin, and B. Vucetic, "Novel nested convolutional lattice codes for multi-way relaying systems over fading channels," in Proc. IEEE Wireless commun. and Networking Conference (WCNC), 2013, pp. 2671-2676.

[6] Z. Xiao, L. Guo, and J. Tracey, "Understanding instant messaging traffic characteristics," in Proc. 27th IEEE Int. Conf. Distributed Computing Systems (ICDCS), 2007, pp. 51-51.

[7] A. Voicila, D. Declercq, F. Verdier, M. Fossorier, and P. Urard, "Lowcomplexity decoding for non-binary LDPC codes in high order fields," IEEE Trans. commun., vol. 58, no. 5, pp. 1365-1375, 2010.

[8] L. Conde-Canencia, E. Boutillon, and A. Al-Ghouwayel, "Complexity comparison of nonbinary LDPC decoders," ICT-MobileSummit, Santander, Spain, Jun. 2009.

[9] B. Rong, T. Jiang, X. Li, and M. Soleymani, "Combine LDPC codes over GF(q) with q-ary modulations for bandwidth efficient transmission," IEEE Trans. Broadcasting, vol. 54, no. 1, pp. 78-84, 2008.

[10] C. Feng, D. Silva, and F. R. Kschischang, "An algebraic approach to physical-layer network coding," IEEE Trans. Inform. Theory, vol. 59, no. 11, pp. 7576-7596, Nov. 2013.

[11] Q. Sun and J. Yuan, "Lattice network codes based on Eisenstein integers," in Proc. 8th IEEE Int. Conf. Wireless and Mobile Computing, Networking and commun. (WiMob), Oct. 2012, pp. 225-231. 